\title{
4 Die Bearbeitung der Quellen im Jalkut Schimoni Zwölfprophetenbuch
}

David Meyer formulierte in seiner Dissertation die These, dass die verwendeten Quellen im Jalkut Schimoni aus einer Vorlage zitiert wurden. Mit seiner Dissertationsschrift verfolgte er das Ziel, den Text von GenR zu finden, der in Jalkut Schimoni zu Genesis für den von ihm ausgewählten Textteil verwendet wurde. ${ }^{1}$ Im Folgenden wird gezeigt, dass dieser Ansatz von Meyer unhaltbar ist. Es wird gezeigt, dass der Autor des Jalkut Schimoni kreativ mit den Quellen verfuhr. Er bearbeitete diese und fügte sie passend in die jeweilige Auslegung ein. Die Möglichkeit, eine „Vorlage“ zu finden, die dem abgebildeten Text der einzelnen Quelle im Jalkut Schimoni zum Zwölfprophetenbuch entspricht, ist nicht möglich. Auch die Verwendung des Jalkut Schimoni als Textressource verlorener Quellentexte ist nicht möglich. In welchen Varianten die Veränderung der Quellen vorgenommen wurde, soll im Folgenden beispielhaft gezeigt werden.

\subsection{Ein Belegvers wird zum auszulegenden Vers}

Der Kommentar zum Zwölfprophetenbuch im Jalkut Schimoni speist sich aus den verschiedensten Quellen aus Talmud und Midrasch. Vorallem bei der verwendeten Midraschliteratur handelt es sich häufig um fortlaufende Auslegungen zu anderen biblischen Büchern. ${ }^{2}$ Diese Auslegungen werden für den Kommentar des Zwölfprophetenbuchs immer dann verwendet, wenn Verse des Zwölfprophetenbuches in der Quelle verarbeitet werden. Diese Verse dienen in dieser Midraschliteratur der Auslegung des jeweiligen biblischen Buches. ${ }^{3}$ Am Beispiel der Auslegung des Jalkut zu Mal 3,19 kann gezeigt werden, wie aus der Quelle GenR 6,6 eine Auslegung zum Vers Mal 3,19 gewonnen wurde. In GenR 6,6 heißt es:

Wohin sind die Sphären der Sonne und des Mondes gesetzt? In den zweiten [Himmel], die Rakia, denn es heißt:

Und Gott gab sie in die Rakia der Himmel. (Gen 1,17)

R. Pinchas sagte im Namen von R. Abbahu: Dieser Vers ist deutlich und die Männer der großen Synagoge erklärten ihn:

1 Vgl. David Théophile Ivan Meyer, La Nature Éditoriale du Yalkut Shimoni. Une étude exploratoire des citations de Bereshit Rabbah XXII,8-10 dans le Yalkut Shimoni (PhD, Universität Leuven), 4-5.

2 Zum Beispiel: Midrasch Samuel, Genesis Rabba, Deuteronomium Rabba oder Sifre Numeri.

3 Zum Beispiel Genesis in Genesis Rabba. 
Du allein bist JHWH. [Du hast den Himmel gemacht und den Himmel der Himmel und all ihr Heer.] (Neh 9,6)

Wohin ist ihr ganzes Heer gegeben worden? In den zweiten [Himmel], die Rakia, das ist die Rakia über dem Himmel. Von der Rakia bis zum Himmel ist es ein Weg von 500 Jahren, und die Breite der Rakia ist ein Weg von 500 [Jahren] und nun siehe, wie hoch die Rakia ist.

Ein Tanna [lehrt]: Am ersten [Tag] des Kreislaufs des Tammuz gibt es keinen Schatten für ein Geschöpf, denn es steht geschrieben:

Und nichts ist vor seiner Glut verborgen. (Ps 19,7)

Ein Tanna [lehrt]: Am ersten Tag des Kreislaufs des Tammuz hat kein Geschöpf einen Schatten, denn es steht geschrieben:

Und nichts ist vor seiner Glut verborgen. (Ps 19,7)

Die Sonnenkugel hat [jedoch] eine Hülle. Was ist der Beleg?

In ihnen hat er dem Sonnenball ein Zelt gesetzt. (Ps 19,5)

Und ein Teich mit Wasser steht davor. Und wenn sie herausgeht, schwächt der Heilige, gepriesen sei er, ihre Kraft im Wasser, damit sie die Welt nicht versengt. Aber in der Zukunft wird Gott sie von ihrer Hülle befreien, um zu verbrennen. Was ist der Beleg?

Und der kommende Tag wird sie verbrennen. (Mal 3,19)

Im Jalkut Schimoni zu Mal 3,19 wird die Kommentierung aus GenR 6,6 wie folgt umgestellt. Die grau hinterlegten Textstellen zeigen die Textumstellungen im Vergleich zur Quelle:

\section{Und der kommende Tag wird sie verbrennen. (Mal 3,19)}

Dies ist es, was die Schrift sagt:

Du bist JHWH allein. Du hast den Himmel und die Himmel der Himmel gemacht. (Neh 9,6)

Wohin sind die Sphären der Sonne und des Mondes gesetzt? In den zweiten [Himmel], die Rakia, denn es heißt:

Und Gott gab sie in die Rakia der Himmel. (Gen 1,17)

R. Pinchas sagte im Namen von R. Abbahu: Dieser Vers ist deutlich und die Männer der großen Synagoge erklärten ihn:

Du allein bist JHWH. [Du hast den Himmel gemacht und den Himmel der Himmel und all ihr Heer]. (Neh 9,6)

Wohin ist ihr ganzes Heer gegeben worden? In den zweiten [Himmel], die Rakia, das ist die Rakia über dem Himmel. Von der Rakia bis zum Himmel ist es ein Weg von 500 Jahren, und die Breite der Rakia ist ein Weg von 500 [Jahren] und nun siehe, wie hoch die Rakia ist.

Ein Tanna [lehrt]: Am ersten [Tag] des Kreislaufs des Tammuz gibt es keinen Schatten für ein Geschöpf, denn es steht geschrieben:

Und nichts ist vor seiner Glut verborgen. (Ps 19,7)

Die Sonnenkugel hat [jedoch] eine Hülle. Was ist der Beleg?

Der Sonne hat er ein Zelt in ihnen gesetzt. (Ps 19,7)

Und vor ihr ist ein Teich mit Wasser. Und in der Stunde, in der sie aufgeht, schwächt der Heilige, gepriesen sei er, ihre Kraft im Wasser, damit sie nicht aufgeht und die Welt verbrennt. Aber in 
der Zukunft wird der Heilige, gepriesen sei er, sie von ihrer Hülle befreien und die Frevler werden verbrennen, denn es heißt:

Und der kommende Tag wird sie verbrennen. (Mal 3,19)

Im Jalkut wird der in GenR 6,6 erwähnte Vers Mal 3,19 der Auslegung zusammen mit Neh 9,6 vorangestellt. Dadurch wird Mal 3,19 zum auszulegenden Vers und verdrängt den eigentlichen Auslegungsvers Gen 1,17 zu einem Belegvers für Mal 3,19.

Eine Quellenverwendung dieser Art findet sich mehrfach im Jalkut Schimoni. 43 Quellenstücke aus GenR ${ }^{4}$ werden so in den Text zur Auslegung des Zwölfprophetenbuches integriert. Es handelt sich also um eine Umstellung die einen neuen Kommentar aus vorhandenem Quellenmaterial schafft, in dem der Text in der Reihenfolge verändert wird. So wird der Auslegungstext zum Vers Gen 1,17 zu einem Auslegungstext zu Mal 3,19. Diese Textumstellungen werden im Jalkut Schimoni vorgenommen ohne diese an entsprechender Stelle kenntlich zu machen.

Ein weiteres Beispiel wie ein Belegvers zum auszulegenden Vers wird, indem mehrere Textteile aus der Quelle gestrichen werden. In Midrasch Samuel 2,1, in der Auslegung zu I Sam 1,11 heißt es wie folgt:

Ich bin JHWH, dein Gott, der dich aus dem Land Ägypten heraufgeführt hat. Mach deinen Mund weit auf, damit ich ihn füllen kann. (Ps 81,11)

R. Chaggai hat im Namen von R. Isaak gesagt: Man verlange nicht Armut am Ort des Reichtums, denn dadurch eilte Hanna zu den Gelübden, denn es heißt:

Und sie legte ein Gelübde ab und sprach. (I Sam 1,11)

Es heißt:

JHWH, ich hörte deinen Ruf, ich fürchtete mich. (Hab 3,2)

Hanna sprach vor dem Heiligen, gepriesen sei er: Herr der Welten! Abraham handelte [nach] deinem Willen, aber du hast ihm [erst] einen Sohn nach hundert Jahr gegeben, [und] Ahab, der Götzendienst trieb, zeugte siebzig Söhne!

Sara handelte [nach] deinem Willen, aber du gabst ihr [erst] einen Sohn nach neunzig Jahren, [und] Isebel, Tochter der Götzenpriester, bekam siebzig Söhne,

denn es steht geschrieben:

Und Ahab hatte siebzig Söhne in Samaria. (II Reg 10,1)

Und wie sie für ihn in Samaria waren, so waren sie für ihn in Jesreel: Jeder einzelne von ihnen hatte Paläste ( $\pi \alpha \lambda \dot{\alpha} \tau \iota$ ) ), einen für den Sommer einen für den Winter.

Dies ist es, was geschrieben steht:

4 Die folgenden GenR Stellen werden im Zwölfprophetenbuch in gleicher Weise verwendet: GenR 14,4; GenR 18,5; GenR 19,9; GenR 23,7; GenR 25,2; GenR 28,5; GenR 28,6; GenR 28,7; GenR 29,3; GenR 31,3; GenR 37,4; GenR 38,6; GenR 42,2; GenR 46,1; GenR 47,5; GenR 49,2; GenR 53,3; GenR 55,5; GenR 56,11; GenR 56,5; GenR 56,9; GenR 59,9; GenR 6,6; GenR 63,12; GenR 66,7; GenR 68,10; GenR 68,9; GenR 69,5; GenR 70,20; GenR 70,6; GenR 71,2; GenR 75,1; GenR 75,8; GenR 78,2; GenR 78,14; GenR 80,2; GenR 80,7; GenR 84,19; GenR 84,5; GenR 85,1; GenR 86,1; GenR 86,2; GenR 93,5. 
Und ich werde das Sommerhaus sowie das Winterhaus schlagen. (Am 3,15)

Nach R. Juda bar Rabbi Simon hatte ein jeder vier, wie es heißt:

Und untergehen sollen die Häuser von Elfenbein. (Am 3,15)

Nach den Gelehrten hatte ein jeder sechs, wie es heißt:

Und verschwinden sollen viele Häuser. (Am 3,15)

Dadurch eilte Hanna zu den Gelübden:

Und sie legte ein Gelübde ab und sprach. (I Sam 1,11)

Für die Auslegung des Verses Hab 3,2 in Jalkut Habakuk wird die Quelle wie folgt verändert, d.h. Textteile gestrichen und damit die Auslegung zu Hab 3,2 extrahiert. In der folgenden Darstellung wird grau hinterlegt gezeigt, welche Textteile im Jalkut übernommen werden:

Ich bin JHWH, dein Gott, der dich aus dem Land Ägypten heraufgeführt hat. Mach deinen Mund weit auf, damit ich ihn füllen kann. (Ps 81,11)

R. Chaggai hat im Namen von R. Isaak gesagt: Man verlange nicht Armut am Ort des Reichtums, denn dadurch eilte Hanna zu den Gelübden, denn es heißt:

Und sie legte ein Gelübde ab und sprach. (I Sam 1,11)

Es heißt:

JHWH, ich hörte deinen Ruf, ich fürchtete mich. (Hab 3,2)

Hanna sprach vor dem Heiligen, gepriesen sei er: Herr der Welten! Abraham handelte [nach] deinem Willen, aber du hast ihm [erst] einen Sohn nach hundert Jahr gegeben, [und] Ahab, der Götzendienst trieb, zeugte siebzig Söhne!

Sara handelte [nach] deinem Willen, aber du gabst ihr [erst] einen Sohn nach neunzig Jahren, [und] Isebel, Tochter der Götzenpriester, bekam siebzig Söhne,

denn es steht geschrieben:

Und Ahab hatte siebzig Söhne in Samaria. (II Reg 10,1)

Und wie sie für ihn in Samaria waren, so waren sie für ihn in Jesreel: Jeder einzelne von ihnen hatte Paläste ( $\pi \alpha \lambda \dot{\alpha} \tau \iota$ ) , einen für den Sommer einen für den Winter.

Dies ist es, was geschrieben steht:

Und ich werde das Sommerhaus sowie das Winterhaus schlagen. (Am 3,15)

Nach R. Juda bar Rabbi Simon hatte ein jeder vier, wie es heißt:

Und untergehen sollen die Häuser von Elfenbein. (Am 3,15)

Nach den Gelehrten hatte ein jeder sechs, wie es heißt:

Und verschwinden sollen viele Häuser. (Am 3,15)

Dadurch eilte Hanna zu den Gelübden:

Und sie legte ein Gelübde ab und sprach. (I Sam 1,11)

Somit ergibt sich ein Auslegungstext der allein auf Hab 3,2 zugeschnitten ist. Dafür werden alle inhatlichen Aspekte, die der Auslegung von I Sam 1,11 dienen gestrichen. Im Jalkut ist diese Auslegung demnach wie folgt abgebildet:

JHWH, ich hörte deinen Ruf, ich fürchtete mich. (Hab 3,2) 


\begin{abstract}
Abraham handelte [nach] deinem Willen, aber du hast ihm [erst] einen Sohn nach hundert Jahr gegeben, [und] Ahab, der Götzendienst trieb, zeugte siebzig Söhne!

Sara handelte [nach] deinem Willen, aber du gabst ihr [erst] einen Sohn nach neunzig Jahren, [und] Isebel, Tochter der Götzenpriester, bekam siebzig Söhne,

denn es steht geschrieben:

Und Ahab hatte siebzig Söhne in Samaria. (II Reg 10,1)

Und wie sie für ihn in Samaria waren, so waren sie für ihn in Jesreel: Jeder einzelne von ihnen hatte Paläste ( $\pi \alpha \lambda \dot{\alpha} \tau \iota$ ) $)$ einen für den Sommer einen für den Winter.

Dies ist es, was geschrieben steht:

Und ich werde das Sommerhaus sowie das Winterhaus schlagen. (Am 3,15)
\end{abstract}

Indem vorhandene Belegverse der Quelle aus dem Text herausgelöst werden und der Quelle vorangestellt werden können Quellentexte verändert werden ohne eigene Wortveränderungen vorzunehmen. Zudem werden Belegverse durch Textstreichungen zu auszulegenden Versen. Der Autor des Jalkut gibt seine Quellen an, greift aber massiv in die Textfolge ein und bildet zudem Quellenstücke nicht vollständig ab.

\title{
4.2 Der Quellentext wird durch eine zweite Quelle ergänzt
}

In Jalkut Schimoni zu Hab 3,1-2 wird eine Quelle (MidrPs 90,2) unterbrochen und durch eine zweite (MidrPs 90,7) ergänzt. Um deutlich zu zeigen, wie der Jalkut Schimoni seine Auslegung konstruiert, werden hier die Quellentexte in der Reihenfolge, in der sie im Jalkut auftreten, hintereinandergestellt (d.h. MidrPs 90,2, MidrPs 90,7, MidrPs 90,2). Die Textteile, die davon im Jalkut verwendet werden, werden grau hinterlegt.

MidrPs 90,2:

Gebet von Mose, dem Mann Gottes. (Ps 90,1) ${ }^{5}$

Unsere Rabbinen und R. Jehuda bar Simon. [Unsere Rabbinen] sagte: Vier ordneten ein Gebet und gebrauchten dabei vor dem Heiligen, gepriesen sei er, zürnende Worte.

1) Jeremia, denn es heißt:

Ich betete zu JHWH, nachdem ich den Kaufbrief übergeben hatte. (Jer 32,16)

Was steht darüber geschrieben, dass er zürnende Worte gebrauchte?

Siehe, die Wälle reichen bis zu der Stadt, sie einzunehmen. (Jer 32,24)

2) Habakuk ordnete ein Gebet, denn es heißt:

Gebet des Propheten Habakuk. (Hab 3,1)

Was steht danach geschrieben?

5 Der Auslegung wird der Vers Hab 3,1 vorangestellt. Während in der Quelle Midrasch Psalm Ps 90,1 ein auszulegender Vers ist, wird dieser im Jalkut nicht übernommen. 
$J H W H$, ich hörte deinen Ruf, ich fürchtete mich. $(\mathrm{Hab} 3,2)^{6}$

3) David ordnete das Gebet, denn es heißt:

Gebet Davids (Ps 17,1)

Was steht gegen oben in Bezug auf die Angelegenheit geschrieben?

Denn du erblickst Mühe und Unmut. (Ps 10,14)

4) Mose ordnete das Gebet, denn es heißt:

Und ich betete zu JHWH und sprach: JHWH verderbe nicht dein Volk. (Dtn 9,26)

Was steht davor?

Warum, JHWH, entbrennt dein Zorn über dein Volk? (Ex 32,11)

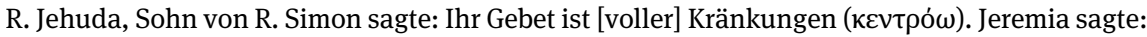

Und ich betete zu JHWH. (Jer 32,16)

Was hat er gesagt?

Gerecht bist du, JHWH, als dass ich mit dir streiten sollte. (Jer 12,1)

Denn ich bin heute, und morgen bin ich nicht, und ich sollte gegen dich streiten?! Und das alles warum? Du bist ja gerecht, als das ich mit dir rechten sollte. Habakuk:

Gebet des Propheten Habakuk. (Hab 3,1)

Was hat er gesagt?

JHWH, ich hörte deinen Ruf, ich fürchtete mich. (Hab 3,2)

David:

Gebet Davids: Höre, JHWH, Gerechtigkeit. (Ps 17,1)

Und was hat er gesagt?

Vor den Leuten ist deine Hand, JHWH. (Ps 17,14)

Mose:

Und ich betete zu JHWH. (Dtn 9,26)

Was hat er gesagt?

Gott, verderbe nicht dein Volk und dein Erbe. (Dtn 9,26)

Das wollen die Worte sagen:

Gebet von Mose, dem Mann Gottes. (Ps 90,1)

MidrPs 90,7:

Gebet von Mose, dem Mann Gottes. (Ps 90,1)

Drei Kinder die kamen, um sich ein Geschenk zu holen. Die einen sagen: Eine Schenkung. Der erste kam und ehrte ihn. Er [der König] fragte ihn: Was willst du? Er antwortete ihm: Ich bitte wegen meines Ungehorsams, dass du mir ein Geschenk gibst. Er gab es ihm. Der zweite kam und er gab es ihm. Als der dritte kam, fragte er ihn: Was willst du? Er antwortete: Ich bitte nicht für mich selbst, sondern für die und die Stadt, welche zerstört ist und die dir gehört, beschließe, dass sie wieder erbaut wird. Der König sagte: Sie sei dir ein großer Kranz! So kam auch David,

6 Anstelle von Hab 3,2 wird im Jalkut Hab 1,3 zitiert: Warum lässt du Falschheit sehen und blickst auf Mühsal? 
um zu beten. Der Heilige, gepriesen sei er, sagte zu ihm: Was willst du? Dass du mein Gebet erhörst, antwortet er, denn es heißt:

Gebet Davids. Höre JHWH, die Gerechtigkeit, erkenne mein Flehen, erhöre mein Gebet. (Ps 17,1)

Habakuk kam und Gott fragte ihn: Was willst du? Er antwortete ihm: Was ich vor dir fälschlich gesprochen habe. Er hatte gesehen, dass Hananja, Mischael und Asarja mitten in den Feuerofen gegangen waren und gerettet wurden. Und er sah, dass R. Chananja ben Teradjon und seine Gefährten im Feuer verbrannt wurden. Als er das sah, rief er: Schande! Er sprach vor ihm: Herr der Welt, diese sind Gerechte und jene sind Gerechte, diese sind Reine und jene sind Reine, diese wurden verbrannt und jene wurden gerettet.

Deshalb ist die Tora erschlafft, und das Recht geht nicht strahlend hervor. (Hab 1,4)

Vielmehr: Dieser Nebukadnezar ist unbeschnitten und unrein, aber Daniel ist heilig und rein und er kleidete Zedekija, der ein Frevler ist, denn es heißt:

Und er veränderte die Kleider seines Gefängnisses. (II Reg 25,29)

Nebukadnezar aber herrschte über Zedekija und kleidete Daniel in Purpur. Pharao ist unbeschnitten und unrein, aber Josef ist heilig und rein, aber der Pharao beherrschte Josef und kleidete ihn mit Byssusgewändern und legte ihm eine goldene Kette um seinen Hals. Ahaschverosch ist unbeschnitten und unrein, aber Mordechai ist heilig und rein. Er [aber] kleidete Mordechai und setzte ihm eine Krone auf, denn es heißt:

Und Mordechai ging von dem König in königlichen Kleidern. (Est 8,15)

Wenn ein Frevler den Gerechten krönt, dann geht das Recht gekrümmt hervor. (Hab 1,4)

Es erschien ihm, der Heilige, gepriesen sei er, und sagte zu ihm: Mir rufst du „Schande“ zu? Steht nicht geschrieben:

Gott der Treue und nicht des Unrechts? (Dtn 32,4)

In diese Stunde sprach er: Ich habe unwissentlich [so] gesprochen, denn es heißt:

Gebet des Propheten Habakuk über Klagelieder. (Hab 3,1)

Mose kam und Gott fragte ihn: Was willst du? Er antwortete: Ich will nichts für mich selbst, sondern für Israel. Dieses, versetzte Gott, ist ein großer Kranz für dich.

Gebet von Mose, dem Mann Gottes. (Ps 90,1)

MidrPs 90,2:

Gebet von Mose, dem Mann Gottes. (Ps 90,1)

Unsere Rabbinen und R. Jehuda bar Simon. [Unsere Rabbinen] sagten: Vier ordneten ein Gebet und gebrauchten dabei vor dem Heiligen, gepriesen sei er, zürnende Worte.

1) Jeremia, denn es heißt:

Ich betete zu JHWH, nachdem ich den Kaufbrief übergeben hatte. (Jer 32,16)

Was steht darüber geschrieben, dass er zürnende Worte gebrauchte?

Siehe, die Bollwerke reichen bis zu der Stadt, sie einzunehmen. (Jer 32,24)

2) Habakuk ordnete das Gebet, denn es heißt:

Gebet für den Propheten Habakuk. (Hab 3,1)

Was steht danach geschrieben?

JHWH, ich hörte deinen Ruf, ich fürchtete mich. (Hab 3,2)

3) David ordnete das Gebet, denn es heißt: 
Gebet Davids (Ps 17,1)

Was steht gegen oben in Bezug auf die Angelegenheit geschrieben?

Denn du erblickst Mühe und Unmut. (Ps 10,14)

4) Mose ordnete das Gebet, denn es heißt:

Und ich betete zu JHWH und sprach: JHWH verderbe nicht dein Volk. (Dtn 9,26)

Was steht davor?

Warum, JHWH, entbrennt dein Zorn über dein Volk? (Ex 32,11)

R. Jehuda, Sohn von R. Simon ( $\Sigma \dot{\prime} \mu \omega v)$ sagte: Ihr Gebet ist [voller] Kränkungen ( $\varepsilon v v \rho o ́ \omega)$. Jeremia sagte:

Und ich betete zu JHWH. (Jer 32,16)

Was hat er gesagt?

Gerecht bist du, JHWH, als dass ich mit dir streiten sollte. (Jer 12,1)

Denn ich bin heute, und morgen bin ich nicht, und ich sollte gegen dich streiten?!

Und das alles warum? Du bist ja gerecht, als das ich mit dir rechten sollte.

Habakuk:

Gebet des Propheten Habakuk. (Hab 3,1)

Was hat er gesagt?

JHWH, ich hörte deinen Ruf, ich fürchtete mich. (Hab 3,2)

David:

Gebet Davids: Höre, JHWH, Gerechtigkeit. (Ps 17,1)

Und was hat er gesagt?

Vor den Leuten ist deine Hand, JHWH. (Ps 17,14)

Mose:

Und ich betete zu JHWH. (Dtn 9,26)

Was hat er gesagt?

Gott, verderbe nicht dein Volk und dein Erbe. (Dtn 9,26)

Das wollen die Worte sagen:

Gebet von Mose, dem Mann Gottes. (Ps 90,1)

Daraus ergibt sich die folgende Auslegung im Jalkut:

\section{Gebet des Propheten Habakuk. (Hab 3,1)}

Die Rabbinen und R. Jehuda und R. Simon ( $(i j \mu \omega v)$ : Die Rabbinen sagten: Vier sind es, die ein

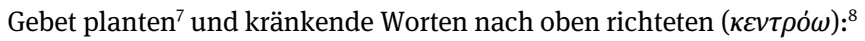

Jeremia:

Da betete ich zu JHWH, nachdem ich den Kaufbrief gegeben hatte. (Jer 32,16)

Was steht nach oben [Kränkendes] geschrieben?

Siehe, die Wälle reichen bis in die Stadt. (Jer 32,24)

Habakuk ordnete ein Gebet:

7 Wörtlich: ordneten.

8 Wörtlich: kränken. 


\section{Gebet des Propheten Habakuk (Hab 3,1)}

Was steht nach oben [Kränkendes] geschrieben?

\section{Warum lässt du $u^{9}$ Falschheit sehen und blickst auf Mühsal? (Hab 1,3)}

Er hatte gesehen, dass Hananja, Mischael und Asarja mitten in den Feuerofen gegangen waren und gerettet wurden. Und er sah, dass R. Chananja ben Teradion und seine Gefährten im Feuer verbrannt wurden. Als er das sah, rief er: Schande! Er sprach vor ihm: Herr der Welt, diese sind Gerechte und jene sind Gerechte, diese sind Reine und jene sind Reine, diese wurden verbrannt und jene wurden gerettet.

Deshalb ist die Tora erschlafft, und das Recht geht nicht strahlend hervor. (Hab 1,4)

Vielmehr: Dieser Nebukadnezar ist unbeschnitten und unrein, aber Daniel ist heilig und rein. Nebukadnezar aber herrschte über Zedekija und kleidete Daniel in Purpur. Ahaschverosch ist unbeschnitten und unrein, aber Mordechai ist heilig und rein. Er [aber] kleidete Mordechai. Pharao ist unbeschnitten und unrein, aber Josef ist heilig und rein, aber der Pharao beherrschte Josef.

Wenn ein Frevler den Gerechten krönt, dann geht das Recht gekrümmt hervor. (Hab 1,4)

Es erschien ihm, der Heilige, gepriesen sei er, und sagte zu ihm: Mir rufst du „Schande“ zu?

Steht nicht geschrieben:

Gott der Treue und nicht des Unrechts? (Dtn 32,4)

In diese Stunde sprach er: Ich habe unwissentlich [so] gesprochen.

\section{Gebet des Propheten Habakuk über Klagelieder. (Hab 3,1)}

David, denn es steht geschrieben:

Das Gebet Davids: Höre, JHWH, die Gerechtigkeit. (Ps 17,1)

Was steht gegen oben in Bezug auf die Angelegenheit geschrieben?

Denn du erblickst Mühe und Unmut. (Ps 10,14)

Mose, denn es steht geschrieben:

Und ich betete zu JHWH. (Dtn 9,26)

Was hat er gesagt?

[Warum, JHWH, entbrennt dein Zorn über dein Volk? (Ex 32,11)

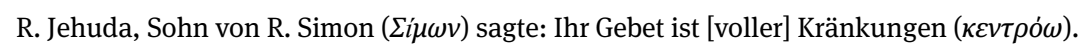
Jeremia sagte:

Und ich betete zu JHWH. (Jer 32,16)

Was hat er gesagt? $]^{10}$

Gerecht bist du, JHWH, als dass ich mit dir streiten sollte. (Jer 12,1)

Denn ich bin heute, und morgen bin ich nicht, und ich sollte gegen dich streiten?!

Und das alles warum? Du bist ja gerecht. Habakuk:

Gebet des Propheten Habakuk. (Hab 3,1)

Was hat er gesagt?

JHWH, ich hörte deinen Ruf, ich fürchtete mich. (Hab 3,2)

David:

9 BH: Warum lässt du mich Falschheit sehen.

10 Das in eckigen Klammern Stehende ist durch Haplographie ausgefallen. 
Gebet Davids: Höre, JHWH, Gerechtigkeit. (Ps 17,1)

Und was hat er gesagt?

Vor den Leuten ${ }^{11}$ ist deine Hand, JHWH. (Ps 17,14)

Mose:

Und ich betete zu JHWH. (Dtn 9,26)

Was hat er gesagt?

Gott, verderbe nicht dein Volk und dein Erbe. (Dtn 9,26)

Dieses Textbeispiel kombiniert die beiden Quellen, indem MidrPs 90,7 in das geteilte Quellenstück MidrPs 90,2 eingefügt wird. Zu Beginn wird Hab 3,1 dem ersten Quellenstück MidrPs 90,2 vorangestellt. Hab 3,1 ersetzt damit den eigentlichen Auslegungsvers der Quelle: Ps 90,1. Der Vers Ps 90,1 wird aus dem Quellenstück entfernt. Zusätzlich wird die Auslegung um ein Teilstück der Quelle MidrPs 90,7 ergänzt. Dafür wird MidrPs 90,2 geteilt und ein ausgewählter Teil der Quelle MidrPs 90,7 eingefügt. Diese „Ergänzungen“ zu MidrPs 90,2 geben weitere Informationen zu Habakuk und erweitern die Auslegung zu Hab 3,1. Das eingefügte Quellenstück aus MidrPs 90,7 passt perfekt in den fortlaufenden Kommentar und führt die Auslegung so nahtlos weiter. Dem Leser zeigt sich auch hier eine fortlaufende Auslegung, die sich durch die getätigten Änderungen argumentativ stark von der Auslegung der ursprünglichen Quelle absetzt. Der Quelltext wird so verändert und für die Auslegung eines anderen Verses (Hab 3,1 anstelle von Ps 90,1) verwendet. ${ }^{12}$ Alle verwendeten Quellenteile werden am Seitenrand angegeben.

\subsection{Verwendung einer Quelle in Teilstücken}

Eine weitere Quellenverwendung im Jalkut ist die Zerteilung einer Quelle in verschiedene Teile, die dann neu kombiniert werden.

Die Auslegungen zu Hos 1,1-6 setzt sich aus den Quellen bPes 87a-b und PRK 24,9 zusammen. Dabei werden die Quellen nicht nacheinander fortlaufend präsentiert, sondern zusammengeschnitten und in ihrer Reihenfolge geändert. Diese Quellenkombination aus Babylonischem Talmud und Pesikta de-Rav Kahana zeigt zudem eine Auslegung, die Quellen aus der babylonischen und der palästinischen Tradition kombiniert. Die folgende Darstellung zeigt die im Jalkut verwendeten Quellen. Die grau hinterlegten Textabschnitte wurden im Jalkut übernommen. Am Seitenrand wird die Position des Quellenstückes, in der Auslegung des Jalkut Schimoni angegeben. Die folgende Darstellung zeigt die verwendeten Quellenstücke aus bPes 87a-b:

11 Siehe Wilhelm Gesenius, Hebräisches und Aramäisches Handwörterbuch über das Alte Testament (18. Aufl., Berlin, Göttingen: Springer, 2013), 763.

12 Das gleiche Prinzip der Quellenverwendung zeigt sich ebenfalls in der Auslegung zu Sach 14,6. Dort wird die Quelle bPes 50a durch die Quelle PRK 4,7 ergänzt. 
WENN FÜR EINE IM HAUSE IHRES MANNES WEILENDE FRAU SOWOHL IHR MANN, ALS AUCH IHR VATER [DAS PESSACHOPFER] GESCHLACHTET HABEN, SO ESSE SIE VON DEM IHRES Mischna MANNES. VERBRACHTE SIE DAS ERSTE FEST IM HAUSE IHRES VATERS, SO ESSE SIE, WENN SOWOHL IHR VATER ALS AUCH IHR MANN FÜR SIE DAS PESSACHOPFER GESCHLACHTET HABEN, WO SIE WILL. EINE WAISE ESSE, WENN [MEHRERE] VORMÜNDER FÜR SIE [DAS PESSACHOPFER] GESCHLACHTET HABEN, WO SIE WILL. EIN SKLAVE ZWEIER BESITZER, DARF [DAS PESSACHOPFER] VON BEIDEN ESSEN. WER ZUR HÄLFTE SKLAVE UND ZUR HÄLFE FREIER IST, DARF VON DEM HERRN NICHT ESSEN.

[Gemara] Hieraus wäre also zu entnehmen, dass es eine fiktive Feststellung gebe? Unter „will“ bPes $87 a$ ist zu verstehen, beim Schlachten. Ich will auf einen Widerspruch hinweisen: Eine Frau esse am ersten Fest vom [Pessachopfer] ihres Vaters, von da an esse sie, wenn sie will, von dem ihres Vaters, und wenn sie will, von dem ihres Mannes!? Das ist kein Widerspruch; dies in dem Falle, wenn es sie [ins Haus ihres Vaters] führt, jenes in dem Falle, wenn es sie nicht führt. So heißt es: Ich war in seinen Augen wie eine, die Frieden findet. (Cant 8,10)

Und R. Jochanan sagte:

Wie es eine junge Frau, die im Hause ihres Schwiegervaters tadelsfrei befunden worden ist, in das Haus ihres Vaters führt, um Lob zu erhalten. Es heißt: An jenem Tage, Spruch JHWHs, wirst du mich „mein Gatte“ nennen und nicht mehr „mein Mann“.

R. Jochanan sagte: Wie eine junge Frau im Hause ihres Schwiegervaters, und nicht wie eine junge Frau im Hause ihres Vaters.

Wir haben eine kleine Schwester noch ohne Brüste. (Cant 8,8)

R. Jochanan sagte:

Das ist Elam, dem es beschieden war zu lernen, nicht aber zu lehren.

Ich bin eine Mauer und meine Brüste Türmen gleich. (Cant 8,10)

R. Jochanan sagte:

Ich bin eine Mauer. (Cant 8,10)

Das ist die Tora;

Meine Brüste Türmen gleich. (Cant 8,10)

Das sind die Schriftgelehrten.

Rava sagte:

Ich bin eine Mauer. (Cant 8,10)

Das ist die Gemeinde Israels.

Meine Brüste Türmen gleich. (Cant 8,10)

Das sind die Bet- und Lehrhäuser.

R. Zutra b. Tobija sagte im Namen Ravs, es heißt:

Unsere Söhne in ihrer Jugend wie großgezogene Pflanzen, unsere Töchter wie Ecksäulen, die nach Tempel-Bauart ausgehauen sind. (Ps 144,12)

Unsere Söhne wie Pflanzen. (Ps 144,12)

Das sind die Jünglinge Israels, die den Geschmack der Sünde nicht gekostet haben.

Unsere Töchter wie Ecksäulen. (Ps 144,12)

Das sind die Töchter Israels, die ihre Pforten für ihre Männer geschlossen halten.

Ebenso heißt es: 
2.Stück im Jalkut ${ }^{13}$

bPes $87 b$
Sie werden voll werden wie die Opferschalen, wie die Ecken des Altars. (Sach 9,15)

Wenn du willst, entnehme man es aus Folgendem:

Unsere Speicher sind voll, Spende gewährend von jeglicher Art. (Ps 144,13)

Die nach Tempel-Bauart ausgehauen sind. Diesen und jenen rechnet es die Schrift an, als wäre der Tempel in ihren Tagen erbaut worden.

Das Wort JHWHs, dass an Hosea erging, in den Tagen Usias, Jotams, Ahas, Hiskias, der Könige von Jehuda. (Hos 1,1)

Vier Propheten weissagten in einer Zeit. Der bedeutendste unter ihnen war Hosea, denn es heißt:

\section{[Am] Anfang war das Wort JHWHs bei Hosea. (Hos 1,2),}

Und da er nicht mit Hosea zu reden anfing, denn von Mose bis Hosea gab es viele andere Propheten, so sagte R. Jochanan: Als erster unter den vier Propheten, die in jener Zeit weissagten. Das sind Hosea, Jesaja, Amos und Micha. Der Heilige, gepriesen sei er, sagte zu Hosea: Israel hat gesündigt! Er hätte zu ihm sagen sollen: Sie sind deine Kinder, sie sind die Kinder, die du liebst. Sie sind die Söhne Abrahams, Isaaks und Jakobs, wende ihnen dein Erbarmen zu.

Nicht genug, dass er dies nicht sagte, sondern er sagte vor ihm: Herr der Welt, die ganze Welt gehört dir. Ersetze sie durch ein anderes Volk.

Der Heilige, gepriesen sei er, sagte: Was mache ich mit diesem Alten machen? Er sagte zu ihm: Nimm dir eine unzüchtige Frau, damit sie dir unzüchtige Kinder gebiert. Und danach sagte er zu ihm: Schicke sie weg! Wenn er sie von seinem Angesicht wegschickt, dann werde auch ich Israel wegschicken. Sofort

sagte JHWH zu Hosea: Nimm dir eine Frau von den Unzüchtigen und Kinder von Unzüchtigen. (Hos 1,2)

Und er ging und nahm Gomer (גומר), die Tochter Diblajims. (Hos 1,3)

Was [bedeutet] „Gomer“?

Rav sagte:

Alle befriedigten (גומרים) sich an ihr.

\section{Tochter Diblajims (דבלים). (Hos 1,3)}

Eine Frau mit schlechtem Ruf (דבה רעה), Tochter einer Frau mit schlechtem Ruf.

Und Samuel sagte:

Alle genießen sie wie einen Feigenkuchen (דבלה).

Und R. Jochanan sagte:

Sie war süß im Mund aller wie ein Feigenkuchen.

Eine andere Auslegung:

Sie begehrten in ihren Tagen, den Reichtum Israels zu zerstören (לגמר).

R. Jochanan sagte:

Sie zerstörten (גמרו) und teilten es ${ }^{14}$ auf, denn es heißt:

Denn König von Aschur ${ }^{15}$ zerstörte sie und verwüstete sie wie den Staub beim Dreschen. (II Reg 13,7)

13 Der hierauf folgende Abschnitt zeigt das an zweiter Stelle stehende Textstück im Jalkut.

14 Israel ist gemeint.

15 BH: von Aram. 
Und sie empfing und gebar [ihm] einen Sohn. Da sagte [JHWH zu ihm]: Nenne seinen Namen Jisreel, denn noch eine kleine [Weile], und ich werde das Blut Jisreels über dem Haus Jehus heimsuchen. Und ich werde dem Königreich des Hauses Israels ein Ende bereiten. (Hos 1,34)

Da wurde sie abermals schwanger und gebar eine Tochter. Da sprach JHWH zu ihm: Nenne sie Nicht-Barmherzigt! Denn fortan will ich dem Haus Israel kein Erbarmen mehr zeigen, dass ich ihnen volle Vergebung gewähren sollte. (Hos 1,6)

Und sie empfing und gebar einen Sohn. [Und er sagte:] Nenne seinen, des Sohnes, ${ }^{16}$ Namen, „[nicht] mein Volk“, denn ihr seid nicht mein Volk. (Hos 1,8-9)

Nachdem ihm zwei Söhne und eine Tochter geboren wurden, sprach der Heilige, gepriesen sei er, zu ihm: Hosea solltest du nicht von deinem Lehrer Mose gelernt haben?! Denn nachdem ich mit ihm gesprochen hatte, trennte er sich von seiner Frau, trenn auch du dich von deiner.

Er sagte vor ihm: Herr der Welt, ich habe Kinder von ihr, und kann sie weder [von mir] scheiden noch wegschicken.

Er sagte zu ihm: Und was bist du, wenn deine Frau unzüchtig ist und deine Kinder unzüchtig sind? Du wirst nicht wissen, ob es deine Kinder sind oder die eines anderen. Ebenso ist [es für mich]: Israel ist mein Kind, sie sind meine geprüften Kinder $^{17}$, Kinder von Abraham, Isaak und Jakob, einer von vier ${ }^{18}$ Besitztümern, die ich in dieser Welt erworben habe.

Eine Erwerbung ist die Tora, wie es heißt:

JHWH erwarb mich als den Anfang seiner Wege. (Prov 8,22)

Eine Erwerbung sind Himmel und Erde, wie es heißt:

Der Himmel und Erde geeignet. (Gen 14,19)

Eine Erwerbung ist der Tempel, wie es heißt:

Dieser Berg, den seine Rechte geeignet. (Ps 78,54)

Eine Erwerbung ist Israel, wie es heißt:

Das Volk, das du dir geeignet hast. (Ex 15,16)

[Und] du sagst zu mir: Ersetze sie durch ein anderes Volk!

Nachdem ihm selbst bewusst geworden war, dass er gesündigt hatte, stand er auf und flehte um Erbarmen für sich.

Der Heilige, gepriesen sei er, sagte zu ihm: Anstatt dass du um Erbarmen für dich bittest, stehe auf und bitte um Erbarmen für die, gegen die ich drei Verfügungen erlassen habe. Er stand auf und flehte um Erbarmen für sie, und er hob alle Verfügungen auf und begann sie zu segnen:

Und die Zahl der Kinder Israels wird sein wie Sand im Meer. Und anstatt dass man zu ihnen gesagt hat: ihr seid nicht mein Volk, wird man zu ihnen sagen: Söhne des lebendigen Gottes. Die Söhne Jehudas und die Söhne Israels werden sich versammeln. (Hos 2,1-3)

Und ich werde es mir im Land einsäen und mich der Nicht-Barmherzigten erbarmen, und zu nicht-mein-Volk sagen: Du bist mein Volk. (Hos 2,25)

16 In BH fehlt: des Sohnes.

17 Marcus Jastrow, A Dictionary of the Targumim, the Talmud Babli and Yerushalmi, and the Midrashic Literature (London: Luzac, 1903), 155: "my tried servants."

18 Im Jalkut sind es drei Besitztümer. Der Tempel wird nicht hinzugezählt. 
1. Stück im Jalkut

\section{Stück im} Jalkut

\section{R. Jochanan sagte:}

Wehe der Herrschaft, die ihre Besitzer begräbt, denn du hast keinen Propheten, der nicht vier Könige besiegt hätte, denn es heisst: ${ }^{19}$

Die Vision, die Jesaja, der Sohn des Amos über Jehuda und Jerusalem empfing. (Jes 1,1)

R. Jochanan sagte:

Warum war Jerobeam, Sohn des Joasch, würdig, zu den Königen des Hauses Davids gezählt zu werden?

Weil er das schlechte Reden über Amos nicht übernahm.

Woher [ist zu belegen], dass er [dazu] gezählt wurde? Weil geschrieben steht:

Wort JHWHs zu Hosea, Sohn des Beeri, in den Tagen Usias, Jotams, Ahas, Hiskias, Könige von Juda, und in den Tagen Jerobeams, Sohn Joaschs, König von Israel. (Hos 1,1)

Und woher ist zu belegen, dass er es nicht annahm? Weil geschrieben steht:

Und Amazia, der Priester von Bet-El, sandte zu Jerobeam, dem König von Israel, um zu sagen: Amos hat sich gegen dich verschworen, inmitten des Hauses Israel. Das Land kann all seine Worte nicht ertragen. Denn so spricht Amos: Jerobeam wird durch das Schwert sterben, und Israel wird aus seinem Land gefangen weggeführt werden. (Am 7,10-11)

Er sagte zu ihm: Gott bewahre, dass der Rechtschaffene dies gesagt hat! Und wenn er es gesagt hat, was sollte ich ihm tun? Die Schechina hat es ihm gesagt.

R. Eleazar sagte ${ }^{20}$ :

Sogar in der Stunde des Zorns des Heiligen, gepriesen sei er, erinnert er sich an Israel, denn es heißt:

Denn ich habe mit dem Haus Israel kein Erbarmen mehr. (Hos 1,6)

R. Jose bar Chanina sagte es von hier:

Denn ich werde ihnen vergeben. (Hos 1,6)

Ferner sagte R. Eleazar: Der Heilige, gepriesen sei er, hat Israel nur deshalb unter die Völker zerstreut, damit Proselyten sich ihnen anschließen, denn es heißt:

Ich will ihn mir in das Land einsäen. (Hos 2,25)

Und man sät eine Sea nur, um viel Kor zu ernten.

R. Jochanan entnimmt es aus Folgendem:

Und der Nicht-Barmherzigten Liebe erweisen. (Hos 2,25)

R. Jochanan sagte im Namen des R. Simon ben Jochai: Es heißt:

Verleumde nicht den Knecht bei seinem Herrn, dass er dir nicht fluche. (Prov 30,10),

Und darauf folgt:

Eine Generation, die ihren Vater flucht und ihre Mutter nicht segnet. (Prov 30,11)

Sollte man denn [einen Knecht] deshalb verleumden, weil [er] seinem Vater flucht und seine Muttern nicht segnet? Vielmehr, selbst in einem Zeitalter, in dem man seinem Vater flucht und seine Mutter nicht segnet, sollst du den Knecht nicht bei seinem Herrn verleumden. Woher ist dies zu belegen? Von Hosea.

19 Bevor Jes 1,1 im Jalkut zitiert wird, wird In den Tagen Usias, Jotams, Ahas, Hiskias. (Hos 1,1) vorangestellt.

20 Im Jalkut wird Hos 1,6 diesem vierten Teil vorangestellt. 
Und Hoschaja sagte: Es heißt:

Die Wohltat seiner Zerstreuung mit Israel. (Jdc 5,11)

Der Heilige, gepriesen sei er, erwies Israel eine Wohltat, indem er sie unter die Völker zerstreute. So sprach einst ein Minäer zu R. Chanina: Wir sind besser als ihr; von euch heißt es:

Denn Joab blieb dort mit ganz Israel sechs Monate, bis er alles, was in Edom männlich war, ausgerottet hatte. (I Reg 11,16)

Wir aber tun euch nichts, wo ihr so viele Jahre bei uns seid. Dieser erwiderte: Wenn du es wünschst, so möge sich dir ein Schüler anschließen. Da schloss sich ihm R. Oschaja an und erwiderte ihm: Weil ihr nicht wisst, wie ihr uns vernichten sollt; [wir] sind ja nicht alle bei euch, und wenn nur diejenigen, die bei euch sind, so würde man euch „verstümmelndes Reich“ nennen. Jener entgegnete: Beim Kapitol zu Rom, damit gehen wir und damit kommen wir.

R. Chija sagte: Es heißt:

Gott kennt den Weg zu ihr, und ihm ist der Platz bekannt. (Ijob 28,23)

Der Heilige, gepriesen sei er, kennt Israel, dass sie die grausamen Verordnungen der Römer nicht ertragen können, daher vertrieb er sie nach Babylonien. Ferner sagte R. Eleazar: Der Heilige, gepriesen sei er, vertrieb nur deshalb Israel nach Babylonien, weil es tief wie die Unterwelt ist, und es heißt:

Aus der Hand der Unterwelt erlöse ich sie, vom Tode kaufe ich sie los. (Hos 13,14)

R. Chanina sagte:

Weil ihre Sprache mit der Sprache der Tora verwandt ist.

R. Jochanan sagte:

Er schickte sie in das Haus ihrer Mutter.

Ein Gleichnis. Wohin schickt jemand seine Frau, wenn er ihr zürnt? In das Haus ihrer Mutter.

Das ist es, was R. Alexandri sagte:

Drei kehrten nach ihrer Heimat zurück, und zwar: Israel, das Geld Ägyptens und die Schrift der Bundestafeln. Israel, wie wir bereits gesagt haben. Das Geld Ägyptens, denn es heißt:

Im fünften Jahre des Königs Rechabeam zog Schischak, der König von Ägypten wider Jerusalem heran. (I Reg 14,25)

Die Schrift der Bundestafeln, denn es heißt:

Ich zerschmettere sie vor euren Augen. (Dtn 9,17)

Und hierzu wird gelehrt, dass die Bundestafeln zerschmettert wurden, während die Buchstaben [in den Himmel] flogen. 
Aus PRK 24,9 wird der folgende Teil verwendet:

3. Stück im Jalkut
Von der Frucht des Mundes wird der Mensch satt des Guten, und das, was Menschenhände getan haben, erlässt man ihm. (Prov 12,14) ${ }^{21}$

Dies steht geschrieben: $:^{22}$

Und Ruben kam zur Grube zurück. (Gen 37,29)

R. Eliezer, R. Jehoschua und die Rabbinen [stritten um diesen Vers].

R. Eliezer sagte:

$\mathrm{Er}^{23}$ war so mit dem in Sack-gekleidet-Sein und dem Fasten auf Grund des Vorfalls [mit Bilha] beschäftigt, dass er nicht frei war [nach Josef zu schauen]. Nachdem er jedoch von diesen ${ }^{24}$ befreit war, kam er und sah in die Grube.

Und siehe, Josef war nicht in der Grube. (Gen 37,29)

R. Jehoschua sagte:

Die Leiden des Hauses wurden auf $i n^{25}$ geladen. Aber nachdem er von dieser Last befreit worden war, kam er und sah in die Grube.

Und siehe, Josef war nicht in der Grube. (Gen 37,29)

Die Rabbinen sagten: Der Heilige, gepriesen sei er, sagte $\mathrm{zu}$ ihm: ${ }^{26} \mathrm{Du}$ batest um die Rückkehr des geliebten Sohnes zu seinem Vater. Bei deinem Leben, ein Sohn deines Sohnes wird Israel zu seinem Vater im Himmel zurückkehren lassen. Und wer war dieser?

Dies war Hosea. Über ihn steht geschrieben:

Wort JHWHs, das Hosea, Sohne des Beeri (בארי) geschah. (Hos 1,1)

Sein Sohn Beera (בארה) (באר), der von Tilgat Pilneeser, dem König von Aschur weggeführt worden war, war ein Fürst der Nachkommen Rubens. (I Chr 5,6)

Warum wird als sein Name hier „Beera“28 genannt?

Weil er ein Erklärer (הארה) der Tora war.

Und Beera (בארה) war die Quelle (בארה) der Tora. Warum starb Beera im Exil? Weil durch sein Verdienst die zehn Stämme zurückkehren werden.

Und warum starb Mose in der Wüste?

Weil durch sein Verdienst die in der Wüste Gestorbenen zurückkehren werden.

R. Berekhja sagte: Der Heilige, gepriesen sei er, sagte zu ihm:

Du warst derjenige, der die Umkehr eröffnet hat. Bei deinem Leben, ein Sohn deines Sohnes wird kommen und der Erste sein, der die Umkehr eröffnen wird:

21 Prov 12,14 wird im Jalkut durch Von der Frucht des Mundes wird der Leib des Mannes gesättigt (Prov 18,20) ersetzt.

22 Im Jalkut heißt es: Das ist Ruben.

23 Ruben.

24 Dem Fasten und in Sack gekleidet sein.

25 Ruben.

26 Im Jalkut heißt es: sagte zu Ruben.

27 Im Jalkut geht dem Vers die Formulierung: „Und es steht geschrieben“ vorraus.

28 Und nicht Beeri. 
Kehre um Israel! (Hos 14,2)

Aus den gezeigten Zuschneidungen und Veränderungen der Reihenfolge ergibt sich dieser fortlaufende Kommentar im Jalkut:

R. Jochanan sagte:

bPes $87 b$

Wehe der Herrschaft, die ihre Besitzer begräbt, denn du hast keinen Propheten, der nicht vier Könige besiegt hätte, denn es heißt:

In den Tagen Usias, Jotams, Ahas, Hiskias. (Hos 1,1)

Und ebenso heißt es:

Vision des Jesaja. (Jes 1,1)

R. Jochanan sagte:

Warum war Jerobeam, Sohn des Joasch, würdig, zu den Königen des Hauses Davids gezählt zu werden?

Weil er das schlechte Reden über Amos nicht übernahm.

Woher [ist zu belegen], dass er [dazu] gezählt wurde? Weil geschrieben steht:

Wort JHWHs zu Hosea, Sohn des Beeri, in den Tagen Usias, Jotams, Ahas, Hiskias, Könige von Juda, und in den Tagen Jerobeams, Sohn Joaschs, König von Israel. (Hos 1,1)

Und woher ist zu belegen, dass er es nicht annahm? Weil geschrieben steht:

Und Amazia, der Priester von Bet-El, sandte zu Jerobeam, dem König von Israel, um zu sagen: Amos hat sich gegen dich verschworen, inmitten des Hauses Israel. Das Land kann all seine Worte nicht ertragen. Denn so spricht Amos: Jerobeam wird durch das Schwert sterben, und Israel wird aus seinem Land gefangen weggeführt werden. (Am 7,10-11)

Er sagte zu ihm: Gott bewahre, dass der Rechtschaffene dies gesagt hat! Und wenn er es gesagt hat, was sollte ich ihm tun? Die Schechina hat es ihm gesagt.

[Am] Anfang war das Wort JHWHs bei Hosea. (Hos 1,2)

bPes $87 a-b$

Der Heilige, gepriesen sei er, sagte zu Hosea: Israel hat gesündigt.

Er hätte zu ihm sagen sollen: Sie sind deine Kinder, sie sind die Kinder, die du liebst. Sie sind die Söhne Abrahams, Isaaks und Jakobs, wende ihnen dein Erbarmen zu.

Nicht genug, dass er dies nicht so sagte, sondern er sagte vor ihm: Herr der Welt, die ganze Welt gehört dir. Ersetze sie durch ein anderes Volk.

Der Heilige, gepriesen sei er, sagte: Was soll ich mit diesem Alten machen? Er sagte zu ihm:

Nimm dir eine unzüchtige Frau, damit sie dir unzüchtige Kinder gebiert. ${ }^{29}$ Und danach sagte er zu ihm: Schicke sie weg! Wenn er sie von seinem Angesicht wegschickt, dann werde auch ich Israel wegschicken. Sofort

sagte JHWH zu Hosea: Nimm dir eine Frau von den Unzüchtigen und Kinder von Unzüchtigen. (Hos

1,2)

Und er ging und nahm Gomer (גומר), die Tochter Diblajims. (Hos 1,3)

Was [bedeutet] „Gomer“?

Rav sagte:

29 Siehe Hos 1,2. 
Alle befriedigten (גומרים) sich an ihr.

Tochter Diblajims (דבלים). (Hos 1,3)

Eine Frau mit schlechtem Ruf (דבה רעה), Tochter einer Frau mit schlechtem Ruf.

Und Samuel sagte:

Alle genießen sie wie einen Feigenkuchen (דבלה).

Und R. Jochanan sagte:

Sie war süß im Mund aller wie ein Feigenkuchen.

Eine andere Auslegung:

Sie begehrten in ihren Tagen, den Reichtum Israels zu zerstören (לגמר).

R. Jochanan sagte:

Sie zerstörten (גמרו) und teilten es ${ }^{30}$ auf, denn es heißt:

Denn König von Aschur ${ }^{31}$ zerstörte sie und verwüstete sie wie den Staub beim Dreschen. (II Reg 13,7)

Und sie empfing und gebar [ihm] einen Sohn. Da sagte [JHWH zu ihm]: Nenne seinen Namen Jisreel, denn noch eine kleine [Weile], und ich werde das Blut Jisreels über dem Haus Jehus heimsuchen. Und ich werde dem Königreich des Hauses Israels ein Ende bereiten. (Hos 1,34)

Und sie empfing und gebar einen Sohn. [Und er sagte:] Nenne seinen, des Sohnes, ${ }^{32}$ Namen, „[nicht] mein Volk“, denn ihr seid nicht mein Volk. (Hos 1,8-9)

Nachdem sie ihm zwei Söhne und eine Tochter geboren hatte, sagte der Heilige gepriesen sei er, zu ihm: Hosea, solltest du nicht von deinem Lehrer Mose gelernt haben?! Denn nachdem ich mit ihm gesprochen hatte, trennte er sich von seiner Frau.

Er sagte vor ihm: Herr der Welt, ich habe Kinder mit ihr, und ich kann sie weder [von mir] scheiden noch wegschicken.

Er sagte zu ihm: Und was bist du, wenn deine Frau unzüchtig ist und deine Kinder unzüchtig sind? Du wirst nicht wissen, ob es deine Kinder sind oder die eines anderen. Ebenso ist [es für mich]: Israel ist mein Kind, sie sind meine geprüften Kinder ${ }^{33}$, Kinder von Abraham, Isaak und Jakob, einer von drei Besitztümern, die ich in dieser Welt erworben habe: Tora, Himmel und Erde und Israel. [Und] du sagst zu mir: Ersetze sie durch ein anderes Volk!

Nachdem ihm selbst bewusst geworden war, dass er gesündigt hatte, stand er auf und flehte um Erbarmen für sich.

Der Heilige, gepriesen sei er, sagte zu ihm: Anstatt dass du um Erbarmen für dich bittest, stehe auf und bitte um Erbarmen für die ${ }^{34}$, gegen die ich drei Verfügungen erlassen habe. $\mathrm{Er}^{35}$ stand auf und flehte um Erbarmen für sie, und $\mathrm{er}^{36}$ hob alle Verfügungen auf und begann sie zu segnen: Und die Zahl der Kinder Israels wird sein wie Sand im Meer. (Hos 2,1)

30 Israel ist gemeint.

31 BH: von Aram.

32 In BH fehlt: des Sohnes.

33 Marcus Jastrow, A Dictionary of the Targumim, the Talmud Babli and Yerushalmi, and the Midrashic Literature (London: Luzac, 1903), 155: "my tried servants."

34 Für Israel.

35 Hosea.

36 Gott. 
Und es steht geschrieben,

Und ich werde es mir im Land einsäen und mich der Nicht-Barmherzigten erbarmen. (Hos 2,25)

Dies ist es, was der Schriftvers sagt:

PRK 24,9

Von der Frucht des Mundes wird der Leib des Mannes gesättigt. (Prov 18,20)

Das ist Ruben: Und Ruben kam zur Grube zurück. (Gen 37,29)

Und der Heilige, gepriesen sei er, sagte zu ihm: Du batest um die Rückkehr des geliebten Sohnes zu seinem Vater. Bei deinem Leben, ein Sohn deines Sohnes wird Israel zu seinem Vater im Himmel zurückkehren lassen. Und wer war dieser? Dies war Hosea. Über ihn steht geschrieben: Wort JHWHs, das Hosea, Sohn des Beeri (בארי), geschah. (Hos 1,1) Und es steht geschrieben:

Sein Sohn Beera (בארה). (I Chr 5,6)

Warum wird als sein Name hier „Beera“37 genannt?

Weil er ein Erklärer (הארה) der Tora war.

Und warum starb Beera im Exil? Weil durch sein Verdienst die zehn Stämme zurückkehren werden.

Und warum starb Mose in der Wüste?

Weil durch sein Verdienst die in der Wüste Gestorbenen zurückkehren werden.

R. Berekhja sagt:

Der Heilige, gepriesen sei er, sagte: Du warst derjenige, der die Umkehr eröffnet hat. Bei deinem Leben, ein Sohn deines Sohnes wird kommen und der Erste sein, der die Umkehr eröffnen wird: Kehre um Israel. (Hos 14,2)

Denn ich habe kein Erbarmen mehr mit der Tochter Israels. (Hos 1,6)

bPes $87 b$

R. Eleazar sagte:

Sogar in der Stunde des Zorns des Heiligen, gepriesen sei er, erinnert er sich an Israel, denn es heißt:

Denn ich habe kein Erbarmen mehr. (Hos 1,6)

R. Jose bar Chanina sagte es von hier:

Denn ich werden ihnen vergeben. (Hos 1,6)

Es können für die Art der Verwendung dieser Quellen mehrere Ergebnisse festgehalten werden: Es werden zwei Quellen gewählt, die die Verse Hos 1,1-6 zitieren. Bei diesen Quellen handelt es sich um eine babylonische und eine palästinische Quelle. Es werden also Auslegungen des Talmuds und eines homiletischen Midrasches (Pesikta de-Rav Kahana) zu einer Auslegung verknüpft. Dafür beginnt der Jalkut mit dem Quellenstück aus bPes 87b, das in der Auslegung des Talmuds an hinterer Stelle steht. Diesem Quellenstück wird eine zweite Passage (bPes 87a-b) aus dem Talmud nachgestellt, die dem ersten Stück in der Quelle vorangestellt ist. Innerhalb dieses Stückes werden mehrere kurze Abschnitte ausgeklammert. Der erste ausgeklammerte Teil stellt sich der Frage, warum es heißt, dass JHWH ,anfing“, mit Hosea (Hos 1,2) zu

37 Und nicht Beeri. 
reden. Diskutiert wird, dass JHWH bereits vorher mit Mose gesprochen hatte und Hosea deshalb nicht der erste war. Es heißt weiter, dass Hosea aber zusammen mit Jesaja, Amos und Micha zu den ersten gehörte, zu denen Gott sprach. Der zweite Teil, der ausgeklammert wird, ist der Teil des Verses Hos 1,6, in dem berichtet wird, dass Hosea eine Tochter geboren wurde.

Im Quellentext wird außerdem von vier Besitztümern gesprochen. Diese sind die Tora, der Himmel und die Erde, Israel und der Tempel. Diese werden im Jalkut auf drei Besitztümer verkürzt, wobei der Tempel nicht erwähnt wird. Alle Belege, die zu den Besitztümern im Talmud angegeben werden, sind ebenfalls im Jalkut nicht zitiert. Zum Ende dieses Quellenstücks wird Hos 2,1-3 verkürzt. Hos 2,25 wird ebenfalls verkürzt wiedergegeben.

Als drittes Quellenstück wird PRK 24,9 eingefügt. Diese Auslegung beginnt mit Prov 12,14. Dieser Vers wird im Jalkut durch Prov 18,20 ersetzt. Nach der Zitation von Gen 37,29 im Jalkut wird ein großes Quellenstück ausgeklammert. Hierbei geht es um verschiedene Meinungen der Rabbinen zur Frage, warum Ruben erst so spät zu Josef zurückkehrte, um ihm zu helfen. Dieser Teil, der sich in keinster Weise auf Hosea bezieht, wird ausgeklammert. Auch die weitere Information, dass Hosea zum Stamm Ruben gehörte, wird so im Jalkut ausgelassen. Auf PRK 24,9 folgt als letztes ein weiteres Stück aus bPes 87b. Dieser Teil steht auch im Talmud an letzter Position und schließt auch im Jalkut die Auslegung ab. ${ }^{38}$

Es zeigt sich, dass der Jalkut Schimoni zwar die angegebenen Quellen benutzte, ihre Veränderung aber stillschweigend durchführt. Diese Vorgehensweise zeigt, dass der Autor kreativ mit den Quellen umgegangen ist. Er verwendet die rabbinische Traditionsliteratur, die er zugeschnitten und in veränderter Reihenfolge der Quellenstücke zu einem eigenen Kommentar zusammenfügt. Diese Art mit Texten zu verfahren, zeigt die Eigenständigkeit des Autors im Umgang mit seinen Vorlagen. Somit kann der Jalkut Schimoni weder als Kompilation, noch als Anthologie bezeichnet werden. Die Anthologie setzt ebenfalls voraus, dass Texte ausgewählt und zusammengestellt werden, diese aber nicht massiv verändert werden. Der Jalkut Schimoni ist vielmehr eine, am fortlaufenden Bibeltext geordnete Neugestaltung der Midraschliteratur, in der, babylonische und palästinische rabbinische Auslegungen zur hebräischen Bibel zu einer Einheit verschmolzen werden.

38 Der im Talmud auf das zitierte Quellenstück folgende Abschnitt wird im Übrigen verwendet, um an späterer Stelle den Vers Hos 2,25 auszulegen. 